\title{
Selective Neural Pathway Targeting Reveals Key Roles of Thalamostriatal Projection in the Control of Visual Discrimination
}

\author{
Shigeki Kato, ${ }^{1}$ Masahito Kuramochi, ${ }^{1,2}$ Kenta Kobayashi, ${ }^{1}$ Ryoji Fukabori, ${ }^{1}$ Kana Okada, ${ }^{1,2}$ Motokazu Uchigashima, ${ }^{3}$ \\ Masahiko Watanabe, ${ }^{3}$ Yuji Tsutsui, ${ }^{4}$ and Kazuto Kobayashi ${ }^{1,2}$ \\ ${ }^{1}$ Department of Molecular Genetics, Institute of Biomedical Sciences, Fukushima Medical University School of Medicine, Fukushima 960-1295, Japan, ${ }^{2}$ Core \\ Research for Evolutional Science and Technology, Japan Science and Technology Agency, Kawaguchi 332-0012, Japan, ${ }^{3}$ Department of Anatomy, Hokkaido \\ University School of Medicine, Sapporo 060-8638, Japan, and ${ }^{4}$ Faculty of Symbiotic Systems Science, Fukushima University, Fukushima 960-1296, Japan
}

The dorsal striatum receives converging excitatory inputs from diverse brain regions, including the cerebral cortex and the intralaminar/ midline thalamic nuclei, and mediates learning processes contributing to instrumental motor actions. However, the roles of each striatal input pathway in these learning processes remain uncertain. We developed a novel strategy to target specific neural pathways and applied this strategy for studying behavioral roles of the pathway originating from the parafascicular nucleus (PF) and projecting to the dorsolateral striatum. A highly efficient retrograde gene transfer vector encoding the recombinant immunotoxin (IT) receptor was injected into the dorsolateral striatum in mice to express the receptor in neurons innervating the striatum. IT treatment into the PF of the vector-injected animals caused a selective elimination of neurons of the PF-derived thalamostriatal pathway. The elimination of this pathway impaired the response selection accuracy and delayed the motor response in the acquisition of a visual cue-dependent discrimination task. When the pathway elimination was induced after learning acquisition, it disturbed the response accuracy in the task performance with no apparent change in the response time. The elimination did not influence spontaneous locomotion, methamphetamine-induced hyperactivity, and motor skill learning that demand the function of the dorsal striatum. These results demonstrate that thalamostriatal projection derived from the PF plays essential roles in the acquisition and execution of discrimination learning in response to sensory stimulus. The temporal difference in the pathway requirement for visual discrimination suggests a stage-specific role of thalamostriatal pathway in the modulation of response time of learned motor actions.

\section{Introduction}

The dorsal striatum in basal ganglia circuitry is the principal structure that mediates learning processes contributing to instrumental motor actions (Knowlton et al., 1996; Hikosaka et al., 2002; Graybiel, 2005; Doyon et al., 2009). Neuronal activity in the dorsal striatum undergoes dynamic changes during the acquisition of instrumental conditioning (Tremblay et al., 1998; Jog et al., 1999; Pasupathy and Miller, 2005). Accumulating evidence

Received Aug. 4, 2011; revised 0ct. 2, 2011; accepted 0ct. 10, 2011.

Author contributions: S.K. and Ka.K. designed research; S.K., M.K., Ke.K., R.F., K.O., M.U., and M.W. performed research; Y.T. contributed unpublished reagents/analytic tools; S.K., M.K., Ke.K., R.F., K.O., M.U., M.W., Y.T., and Ka.K. analyzed data; S.K. and Ka.K. wrote the paper.

This work was supported by grants-in-aid from the Ministry of Education, Science, Culture, and Sports of Japan and by Core Research for Evolutional Science and Technology of Japan Science and Technology Agency. A part of this work was supported by "Highly Creative Animal Model Development for Brain Sciences," performed under the Strategic Research Program for Brain Sciences by the Ministry of Education, Culture, Sports and Technology of Japan. We thank Dr. I. Pastan for providing anti-Tac(Fv)-PE38 and St. Jude Children's Research Hospital (Dr. A. Nienhuis) and George Washington University for providing the HIV-1-based vector system. We are grateful to M. Kikuchi, N. Sato, M. Watanabe, and T. Kobayashi for technical support in the animal experiments.

The authors declare no competing financial interests.

Correspondence should be addressed to Dr. Kazuto Kobayashi, Department of Molecular Genetics, Institute of Biomedical Sciences, Fukushima Medical University School of Medicine, Fukushima 960-1295, Japan. E-mail: kazuto@fmu.ac.jp.

DOI:10.1523/JNEUROSCI.4005-11.2011

Copyright $\odot 2011$ the authors $\quad 0270-6474 / 11 / 3117169-11 \$ 15.00 / 0$ from lesion and pharmacological studies indicates the important roles of the dorsal striatum in different paradigms for goaldirected and habitual actions (Packard and Knowlton, 2002; Balleine et al., 2009), along with the functional heterogeneity in the dorsolateral and dorsomedial subdivisions of the striatum for these behaviors (Rogers et al., 2001; Yin et al., 2004, 2006; Yin, 2010). Anatomically, the striatum receives converging excitatory inputs from many cortical areas and the intralaminar/midline thalamic nuclei and projects to the basal ganglia output nuclei, which form the feedback loop to the cortical areas via various thalamic nuclei (Alexander and Crutcher, 1990; DeLong, 1990; Parent and Hazrati, 1995).

The intralaminar thalamic nuclei contain multiple nuclear groups, including the centromedian nucleus $(\mathrm{CM})$ and the parafascicular nucleus (PF) in primates or the PF in rodents (Smith et al., 2004, 2009). These groups provide the major sources of thalamostriatal inputs and extend innervations to specific subdivisions of the striatum (Berendse and Groenewegen, 1990; François et al., 1991). Studies with single-unit recording and pharmacological inhibition of neural activity suggest that the $\mathrm{CM} / \mathrm{PF}$ neurons in monkeys participate in the processes of attention and arousal triggered by sensory events (Matsumoto et al., 2001; Minamimoto and Kimura, 2002; Kimura et al., 2004). 
Studies on the behavioral role of the intralaminar thalamic nuclei in rodents have been performed by the use of several types of memory and learning paradigms (Burk and Mair, 1998; MassanésRotger et al., 1998; Mair et al., 2002). However, little is known about the specific roles of the thalamostriatal pathways derived from the intralaminar nuclear groups in the behavioral processes linked to basal ganglia function.

We addressed the roles of the thalamostriatal pathway in learning processes, focusing on the neural pathway arising from the PF and innervating the dorsolateral striatum in mice. We applied immunotoxin (IT)-mediated cell targeting, which is a genetic approach to ablate specific cell types from a complex neural circuitry (Kobayashi et al., 1995; Sano et al., 2003; Yasoshima et al., 2005), for selective elimination of neural pathways in the brain. Human interleukin-2 receptor $\alpha$-subunit (IL-2R $\alpha$ ), a receptor molecule for the recombinant IT, was expressed in the neurons innervating the striatum in mice by using a highly efficient retrograde gene transfer (HiRet) vector (Kato et al., 2011). Treatment of the PF with IT in the HiRet vector-injected animals induced a selective, efficient elimination of the thalamostriatal pathway. We tested the behavioral consequence of this pathwayspecific elimination with different paradigms that require the function of the dorsal striatum. Our results demonstrate the roles of the thalamostriatal pathway derived from the PF in the acquisition and performance of a visually cued discrimination task.

\section{Materials and Methods}

Animals. Male C57BL/6J mice at the age of 8-12 weeks were used in the present study. The mice were maintained at $22 \pm 2^{\circ} \mathrm{C}, 60 \%$ humidity, and a $12 \mathrm{~h} \mathrm{light/dark}$ cycle with food and water available ad libitum. For operant conditioning tasks, food feeding was controlled to maintain the mice at $85 \%$ of their ad libitum weight. Animal care and handling procedures were conducted in accordance with the guidelines established by the Experimental Animal Center of Fukushima Medical University.

Viral vector preparation. Fusion glycoprotein B type (FuG-B) was composed of the extracellular and transmembrane domains of the glycoprotein derived from the rabies virus challenged virus standard (CVS) strain and the cytoplasmic domain of the vesicular stomatitis virus glycoprotein (Kato et al., 2011). A part containing the domains of the rabies virus glycoprotein in FuG-B cDNA was exchanged with the corresponding part derived from the rabies virus Pasteur virus (PV) strain (termed FuG-B2). The envelope plasmid for the HiRet vector contained FuG-B or FuG-B2 cDNA under the control of cytomegalovirus enhancer/chicken $\beta$-actin promoter (pCAGGS-FuG-B or pCAGGS-FuG-B2). The transfer plasmids contained the cDNA encoding enhanced green fluorescent protein (GFP) or IL-2R $\alpha$ fused to GFP (IL-2R $\alpha-$ GFP) downstream of the murine stem cell virus promoter.

DNA transfection and viral vector preparation were performed as described previously (Hanawa et al., 2002, 2004) with some modifications. HEK293T cells were transfected with transfer, envelope, and packaging plasmids by the calcium phosphate precipitation method. Viral vector particles were pelleted by centrifugation at $6000 \times g$ for $16-18 \mathrm{~h}$ and resuspended in PBS. The particles were then applied to a Sepharose Q FF ion-exchange column (GE Healthcare) in PBS and eluted with a linear $0.0-1.5 \mathrm{M} \mathrm{NaCl}$ gradient. The fractions were monitored at absorbance $260 / 280 \mathrm{~nm}$. The peak fractions containing the particles were collected and concentrated by centrifugation through a Vivaspin filter (Vivascience). Proper concentrations of viral vectors were used for transduction of HEK293T cells, and functional titer (transduction unit) was measured by flow cytometry (FACSCalibur; BD Biosciences). For determination of the RNA titer, viral RNA in vector preparations was isolated with a NucleoSpin RNA virus kit (Clontech), and the copy number of the RNA genome was determined by using a Lenti-X quantitative reverse transcription (qRT)-PCR titration kit (Clontech). PCR amplification was performed on duplicate samples by using a StepOne real-time PCR sys- tem (Applied Biosystems) under the following conditions: one cycle of $95^{\circ} \mathrm{C}$ for $3 \mathrm{~min}$; and 40 cycles of $95^{\circ} \mathrm{C}$ for $15 \mathrm{~s}$ and $54^{\circ} \mathrm{C}$ for $1 \mathrm{~min}$.

Intracranial surgery. Mice were anesthetized with sodium pentobarbital $(50 \mathrm{mg} / \mathrm{kg}$, i.p.), and vectors were introduced into the dorsolateral region of the striatum $(1.0 \mu \mathrm{l} / \mathrm{site}$, four sites) through a glass microinjection capillary connected to a microinfusion pump (ESP-32; Eicom). The functional titers of the vector solution were $2.0 \times 10^{9}$ transduction units/ml for the HiRet-IL-2R $\alpha$-GFP vector and $1.0 \times 10^{9}$ transduction units/ml for the HiRet-GFP vector. The functional titers were adjusted to achieve a similar level of gene transfer efficiency into the PF between these two vectors. The anteroposterior, mediolateral, and dorsoventral coordinates from bregma and dura were as follows (in $\mathrm{mm}$ ): 1.0/2.5/2.0 (site 1), 1.0/2.5/2.7 (site 2), 0/2.0/2.0 (site 3), and 0/2.0/2.7 (site 4) according to an atlas of the mouse brain (Paxinos and Franklin, 2001). Injection was performed at a constant flow rate of $0.2 \mu \mathrm{l} / \mathrm{min}$.

For IT injection, anti-Tac(Fv)-PE38 (Kreitman et al., 1994) was diluted to a final concentration of $80 \mu \mathrm{g} / \mathrm{ml}$ in PBS containing $0.1 \%$ mouse serum albumin. The IT solution or PBS was injected at a constant flow speed of $0.2 \mu \mathrm{l} / \mathrm{min}$ into the PF $(0.4 \mu \mathrm{l} /$ site, two sites $)$ through a glass micropipette connected to the microinfusion pump. The anteroposterior, mediolateral, and dorsoventral coordinates from bregma and dura were as follows (in mm): $-2.1 / 0.7 / 3.0$ (site 1 ) and $-2.4 / 0.7 / 3.0$ (site 2).

For retrograde axonal labeling, cholera toxin subunit $\mathrm{B}$ conjugated to Alexa Fluor 555 (Invitrogen) was dissolved in a final concentration of 0.2 $\mathrm{mg} / \mathrm{ml}$ in PBS. Solution containing the conjugated cholera toxin subunit $\mathrm{B}$ was injected at a constant velocity of $0.1 \mu \mathrm{l} / \mathrm{min}$ into the dorsolateral striatum ( $0.5 \mu \mathrm{l} /$ site, four sites) with the microinfusion pump by using the same coordinates as used for the vector injection. For anterograde axonal labeling, solution containing $10 \%$ biotinylated dextran amine (BDA) (molecular weight, 10,000; Invitrogen) was injected into the PF $(0.1 \mu \mathrm{l} /$ site, one site) by using the following coordinates (in $\mathrm{mm}$ ): anteroposterior, -2.2; mediolateral, 0.7; and dorsoventral, 3.0 from bregma and dura at a constant velocity of $0.025 \mu \mathrm{l} / \mathrm{min}$.

Histology. Mice were anesthetized with sodium pentobarbital and perfused transcardially with PBS followed by fixation with $4 \%$ paraformaldehyde in $0.1 \mathrm{~m}$ phosphate buffer, $\mathrm{pH}$ 7.4. Sections ( $30 \mu \mathrm{m}$ thick) were incubated with a primary antibody for GFP (rabbit, 1:2000; Invitrogen) and then with a biotinylated secondary antibody (anti-rabbit IgG, 1:500; Jackson ImmunoResearch). The immunoreactive signals were visualized by use of a Vectastain Elite ABC kit (Vector Laboratories) with 3,3'diaminobenzide tetrahydrochloride $/ \mathrm{H}_{2} \mathrm{O}_{2}$ as a chromogen. For double fluorescence detection of GFP expression and cholera toxin subunit $\mathrm{B}$ labeling, sections were incubated with anti-GFP antibody (rabbit, 1:2000) and then with a secondary antibody conjugated to fluorescein isothiocyanate (anti-rabbit IgG, 1:500; Jackson ImmunoResearch). Fluorescent images were taken under a confocal laser-scanning microscope equipped with proper filter cube specifications. For anterograde axonal labeling, the BDA signals were visualized with the Vectastain Elite ABC kit and nickel ammonium sulfate. For cell counts, a series of sections through the PF were used for immunostaining. The number of stained cells was counted with a computer-assisted imaging program (NIH Image 1.62). Eight to 10 sections obtained from individual animals were used for cell counts, and the average per section was calculated.

Behavioral analysis. Locomotor activity was measured with a movement analyzer equipped with photobeam sensors (SV-10; Toyo Sangyou). The number of beam breaks was counted for every $5 \mathrm{~min}$ session. The total number of beam breaks in a $30 \mathrm{~min}$ test period was calculated to evaluate the spontaneous locomotor activity during the pretreatment (sessions -6 to -1 ) and the drug-induced locomotor activity after methamphetamine (METH) treatment (sessions 2-7).

For the rotarod test, mice were placed on an accelerating rotarod (ENV-575M; Med Associates), and the rotation speed was increased from 4 to $40 \mathrm{rpm}$ over $3 \mathrm{~s}$ in each trial. The mice were trained three trials per day with a $30 \mathrm{~min}$ intertrial interval for 3 consecutive days. The time that each mouse maintained its balance on the rotating rod was measured as latency to fall.

The two-choice reaction time, simple reaction time, and progressive ratio tasks were conducted in an operant chamber $(21.6 \times 17.8 \times 12.7$ $\mathrm{cm}$ ) equipped with a pellet receptacle in the center of a front panel 

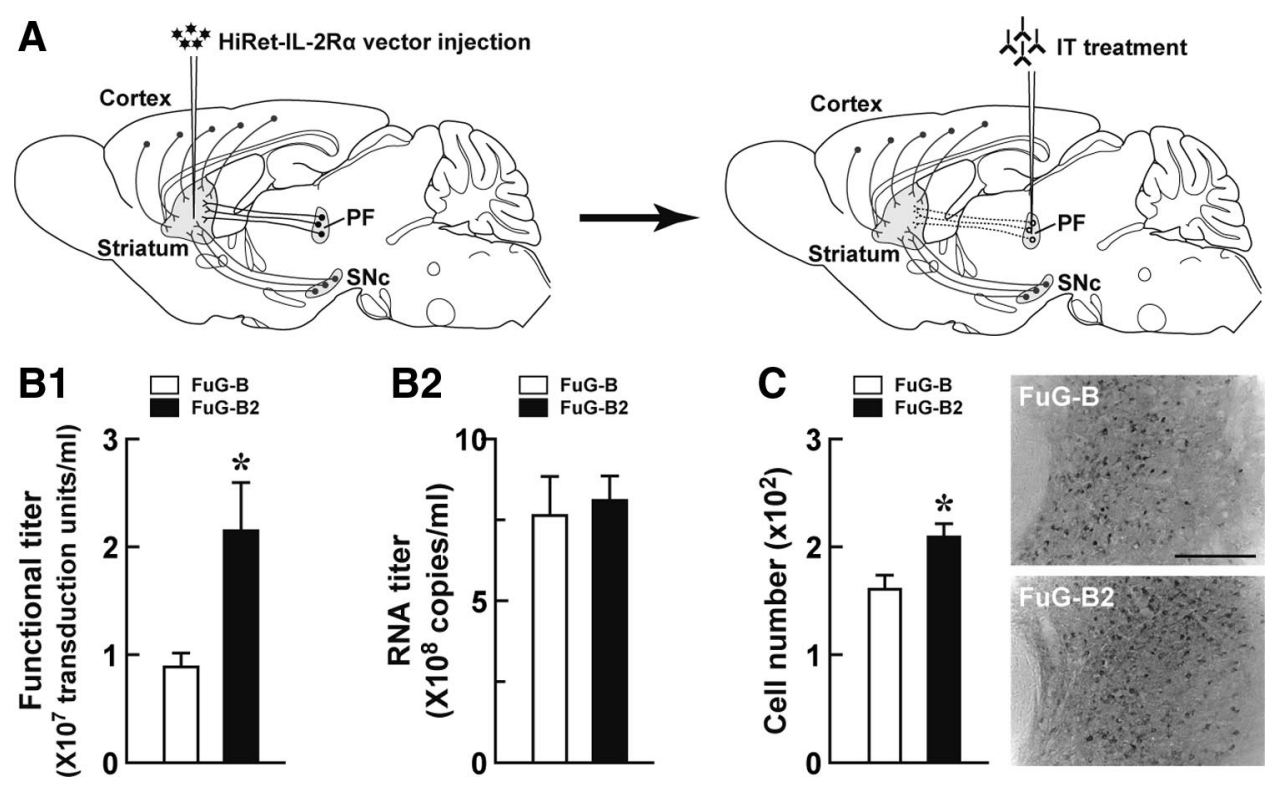

Figure 1. Strategy for selective neural pathway targeting and improvement of the gene transfer efficiency of the HiRet vector. $A$, Selective targeting of the thalamostriatal pathway. Injection of the HiRet vector encoding human IL-2R $\alpha$ into the dorsolateral striatum causes expression of transgene products in various neural pathways innervating the striatum. Subsequent treatment by injection of recombinant IT into the PF of the vector-injected animals induces selective elimination of the neural pathway arising from the PF and innervating the striatum. Dotted lines indicate the removal of the targeted neural pathway. $\boldsymbol{B}$, Titration of the pseudotyped vectors. HEK293T cells in 1810 - $\mathrm{cm}$ tissue culture dishes were transfected with the envelope plasmid encoding FuG-B2 or FuG-B together with the transfer plasmid encoding IL-2R $\alpha-G F P$ and the packaging plasmids. Vector particles were pelleted by centrifugation and resuspended in $1 \mathrm{ml}$ of PBS. HEK293T cells were transduced with proper concentrations of viral vectors, and the functional titer was determined by flow cytometry $\left(\boldsymbol{B}_{\boldsymbol{1}}\right)$. The RNA titer in vector preparations was measured by qRT-PCR analysis $\left(\boldsymbol{B}_{2}\right)$. Values were obtained from four independent experiments. ${ }^{*} p<0.05$, significant difference from the functional titer of the FuG-B vector (Student's $t$ test). $C$, In vivo gene transfer of the pseudotyped vectors. Viral vectors with FuG-B or FuG-B2 with equivalent copy numbers of viral RNA $\left(1.2 \times 10^{10}\right.$ copies $/ \mathrm{ml}, 1.0 \mu \mathrm{l}$ at four sites) were injected into the dorsolateral striatum of mice ( $n=4$ for each group). Five weeks later, sections through the PF were used for immunohistochemistry with anti-GFP antibody. The left shows the number of GFP-positive cells per section, and the right indicates representative images of transgene expression in the PF. ${ }^{*} p<0.05$, significant difference from the cell number of the FuG-B vector (Student's $t$ test). Scale bar, $200 \mu \mathrm{m}$.

(ENV-307W; Med Associates). Two retractable levers were mounted at either side of the receptacle, and a stimulus light was placed above each lever. The chamber was enclosed in a sound-attenuating box and illuminated with a house light during the trials. The mice were shaped to press the levers to obtain the delivery of a $20 \mathrm{mg}$ food pellet (Bioserv Biotechnologies).

For the two-choice reaction time task, the conditioning schedule was composed of three continuous phases. In the first phase, the mice were trained to press one of the two levers (left or right) randomly presented for food pellet delivery. In the second phase, the animals were reinforced to press the lever used for training during the first phase within $5 \mathrm{~s}$ after the initiation of lever presentation. For the third phase, the two levers were presented at the same time and either of the levers was randomly illuminated with the stimulus light. The mice were required to press the lever on the illuminated side for the reward within a $5 \mathrm{~s}$ test period after the onset of lever presentation. If the lever-press responses were emitted, the lever was retracted and the trial was terminated. The initiation of each trial was signaled by a $3 \mathrm{~s}$ acoustic stimulus, and the intertrial interval was 20 s. Each session was ended when 40 rewards were earned or when 30 min had elapsed. When the mice earned 150 rewards in the first phase, they advanced to the second phase. When the mice earned the same number of rewards during the second phase, they moved to the third phase.

For the simple reaction time task, the conditioning schedule consisted of two serial phases. In the first phase, only one lever illuminated by the stimulus light was presented and mice were trained to press the lever contingent on food delivery. In the second phase, the mice were reinforced to press the lever within a $5 \mathrm{~s}$ test period after the lever presentation. If the lever-press responses were evoked, the lever was retracted and the trial was terminated. The initiation of each trial was signaled by a $3 \mathrm{~s}$ acoustic stimulus, and the intertrial interval was $20 \mathrm{~s}$. Each session was ended when 40 rewards were earned or when 30 min had elapsed. When the mice earned 150 rewards during the first phase, they advanced to the second phase.

For the progressive ratio task, mice were trained by using a fixed ratio 1 schedule for four consecutive daily sessions and then a fixed ratio 4 schedule for six consecutive daily sessions. In the fixed ratio 1 schedule, each lever press was reinforced with the delivery of a food pellet, and in the fixed ratio 4 schedule, every fourth lever press was reinforced by the same reward. Each session lasted $30 \mathrm{~min}$. The mice were tested to make a criterion number of lever presses for the food delivery. The criterion was set at two lever presses for the first trial and then doubled with each successive trial. A test session ended after $2 \mathrm{~h}$ or when the mice had made no response during a 3 min period.

Statistical analysis. For statistical comparisons, the ANOVA, the post hoc Bonferroni's test, and Student's $t$ test were used with significance set at $p<0.05$. All values were expressed as the mean \pm SEM of the data.

\section{Results}

HiRet vector drives gene expression in striatal input pathways We developed a novel strategy for conditional targeting of specific neural pathways in the brain and used this strategy for selective elimination of the thalamostriatal pathway (Fig. 1A). First, the HiRet vector encoding human IL-2R $\alpha$ is injected into the dorsolateral striatum to express the receptor in the neural pathways innervating the striatum. The HiRet vector is a pseudotype of human immunodeficiency virus type 1-based vector with FuG-B consisting of the extracellular and transmembrane domains of rabies virus glycoprotein and the cytoplasmic domain of vesicular stomatitis virus glycoprotein. This vector enables highly efficient delivery of a transgene into neurons through retrograde axonal transport (Kato et al., 2011). Subsequently, the PF of the HiRet vector-injected animals is 
treated with the recombinant IT. IT recognizes the neurons harboring IL-2R $\alpha$ and induces selective removal of the targeted neural pathway.

In the present study, we produced the HiRet vector pseudotyped with a variant of FuG-B (termed FuG-B2), in which the extracellular and transmembrane domains of the glycoprotein derived from the rabies virus CVS strain were replaced by the counterparts of the glycoprotein derived from the rabies virus PV strain. We compared the property of the HiRet vectors pseudotyped with FuG-B and FuG-B2, encoding IL-2R $\alpha-$ GFP as a transgene. The functional titer (transduction unit) in vector preparations was measured by flow cytometry (Fig. $1 B_{1}$ ). The titer of the FuG-B2-pseudotyped vector was increased to 2.4 times greater than that of the FuG-B pseudotype $\left(t_{(6)}=2.76, p<\right.$ $0.05)$. The RNA titer in vector preparations, which represents the concentration of physical particles of the vector, was determined by qRT-PCR analysis (Fig. $1 B_{2}$ ). The RNA titer showed no significant difference between the FuG-B and FuG-B2 pseudotypes $\left(t_{(6)}=0.34, p=0.75\right)$. The FuG-B2-pseusdotyped vector thus improved efficiency of gene transduction with no apparent influence on the yield of vector particles. To evaluate the in vivo gene transfer through retrograde transport, we injected the vector pseudotyped with FuG-B or FuG-B2 with an equivalent RNA titer of $1.2 \times 10^{10}$ copies $/ \mathrm{ml}(1.0 \mu \mathrm{l}$ at four sites $)$ into the dorsolateral striatum of mice. Sections through the PF were immunostained with anti-GFP antibody, and the number of GFP-positive cells was counted. This number was elevated in the FuG-B2 pseudotype-treated striatum above that in the FuG-B pseudotype-treated one $\left(t_{(6)}=2.71, p<0.05\right)$, showing a 1.3 -fold elevation (Fig. 1C). These results demonstrate that the FuG-B2 pseudotype enhanced not only the gene transfection of cultured cells but also the in vivo gene transfer through retrograde transport. We thus used the HiRet vector with this new fusion glycoprotein for subsequent experiments.

To induce the expression of human IL-2R $\alpha$ in the thalamostriatal pathway arising from the PF and innervating the dorsolateral striatum, we injected the HiRet vector encoding human IL-2R $\alpha$-GFP (HiRet-IL-2R $\alpha$-GFP, $2.0 \times 10^{9}$ transduction units $/ \mathrm{ml}$ ) into the striatum of mice by stereotaxic surgery and prepared brain sections at 5 weeks after the viral injection. GFP immunohistochemistry of sections through the striatum showed that the vectors had transduced neurons in a large area covering the dorsolateral striatum (Fig. 2A). Cresyl violet staining and immunostaining for the neuronal marker $\mathrm{NeuN}$ indicated no cell damage on the injected side of the striatum (Fig. $2 B$ ). GFP immunohistochemistry of sections through the primary motor cortex (M1), the primary somatosensory cortex (S1), the substantia nigra pars compacta $(\mathrm{SNc})$, and the PF revealed IL-2R $\alpha$-GFP expression in these brain regions that had occurred via retrograde transport (Fig. 2C). To estimate the efficiency of retrograde gene transfer into the $\mathrm{PF}$, we injected the retrograde tracer cholera toxin subunit $B$ conjugated to Alexa Fluor 555 into the dorsolateral striatum of the HiRet-IL-2R $\alpha-$ GFP vector-injected mice and then performed double fluorescence histochemistry (Fig. 2D). Expression of the transgene was detected in the majority of PF neurons labeled by cholera toxin subunit $\mathrm{B}(82.8 \pm 2.2 \%, n=3)$.

\section{IT treatment selectively eliminates thalamostriatal pathway}

Mice received a bilateral injection of the HiRet-IL-2R $\alpha-$ GFP or HiRet-GFP vector, and 5 weeks later they were unilaterally injected with IT or PBS into the PF by stereotaxic surgery. On day 7 after IT/PBS treatment, the brains were processed, and sections were prepared for histochemistry. Sections through the PF were stained by GFP immunohistochemistry, and the number of GFPpositive cells was then counted (Fig. $3 A_{1}$ ). Evidence for a loss of immunopositive cells was found only on the IT-treated side of the HiRet-IL-2R $\alpha$-GFP vector-injected mice. One-way ANOVA showed a significant difference among the four mouse groups $\left(F_{(1,12)}=116.75, p<0.001\right)$, and the cell number of the HiRetIL-2R $\alpha-$ GFP/IT group was remarkably reduced compared with that of each of the HiRet-GFP/PBS, HiRet-GFP/IT, and HiRetIL-2R $\alpha-$ GFP/PBS groups ( $p<0.001$, Bonferroni's test). Cresyl violet staining of the $\mathrm{PF}$ sections indicated no grossly visible tissue damage on the IT-treated side (Fig. $3 A_{2}$ ). GFP immunohistochemistry showed a normal localization of immunopositive cells in the M1, S1, and SNc on the IT-treated side (Fig. $3 A_{3}$ ), indicating intactness of the corticostriatal and nigrostriatal pathways. In addition, GFP immunostaining on the same side also displayed a normal distribution of immunopositive cells in the central lateral nucleus (CL), which is another nucleus innervating the dorsolateral striatum in the intralaminar thalamic nuclear complex (Fig. $3 A_{4}$ ). The data indicate that the CL-derived thalamostriatal pathway was unaffected by the IT injection. To examine the distribution of synaptic terminals arising from the PF, we performed anterograde axonal tracing by injecting $\mathrm{BDA}$ into the $\mathrm{PF}$ of the HiRet-IL-2R $\alpha$-GFP vector-injected mice, in which the PF region was unilaterally treated with IT or PBS. BDA staining revealed a large number of the labeled synaptic terminals in the dorsolateral striatum on the PBS-treated side, whereas the number of the terminals on the IT-treated side was profoundly decreased (Fig. 3B). These results demonstrate that the IT treatment caused a selective elimination of the PF-derived thalamostriatal pathway in the HiRet-IL-2R $\alpha$-GFP vectorinjected animals.

We investigated the time course of cell elimination after IT treatment. Mice that had received an injection of the HiRet-IL$2 \mathrm{R} \alpha-\mathrm{GFP}$ vector were unilaterally injected with PBS or IT into the PF. Sections were prepared on different days after the treatment and used for GFP immunostaining (Fig. 3C). The number of GFP-positive cells displayed a significant difference between the two groups (group effect, $F_{(1,6)}=80.73, p<0.001$; day effect, $F_{(4,24)}=11.55, p<0.001$; group $\times$ day interaction, $F_{(4,24)}=$ $10.73, p<0.001$, two-way ANOVA), showing a gradual reduction in the IT-treated group $(p<0.001$, Bonferroni's test). The cell number in this group reached a plateau by day 7 , and the reduced number was still sustained by day 9 .

\section{Thalamostriatal elimination does not alter locomotion and motor skill learning}

The dorsal striatum is involved in spontaneous locomotion and motor activation in response to systemic administration of drugs that stimulate dopamine release (Costall et al., 1976; Coyle and Schwarcz, 1976). We tested the motor functions of mice that lacked the thalamostriatal pathway arising from the PF. Mice were given an injection of the HiRet-IL-2R $\alpha$-GFP or HiRet-GFP vector into their dorsolateral striatum and then were injected with IT or PBS solution into the PF. On day 7 after the IT/PBS injection, the mice underwent behavioral analysis. We monitored spontaneous locomotion and motor activation after administration of METH (4 mg/kg, i.c.) in the open field (Fig. $4 A$ ). Neither spontaneous locomotor activity $(30 \mathrm{~min})$ nor METH-induced locomotor activity $(30 \mathrm{~min})$ displayed a marked difference among the HiRet-IL-2R $\alpha-$ GFP and HiRet-GFP vector-injected mice that received IT or PBS treatment $\left(F_{(3,24)}=0.59, p=0.63\right.$ for spontaneous locomotor activity; $F_{(3,24)}=0.18, p=0.91$ for 

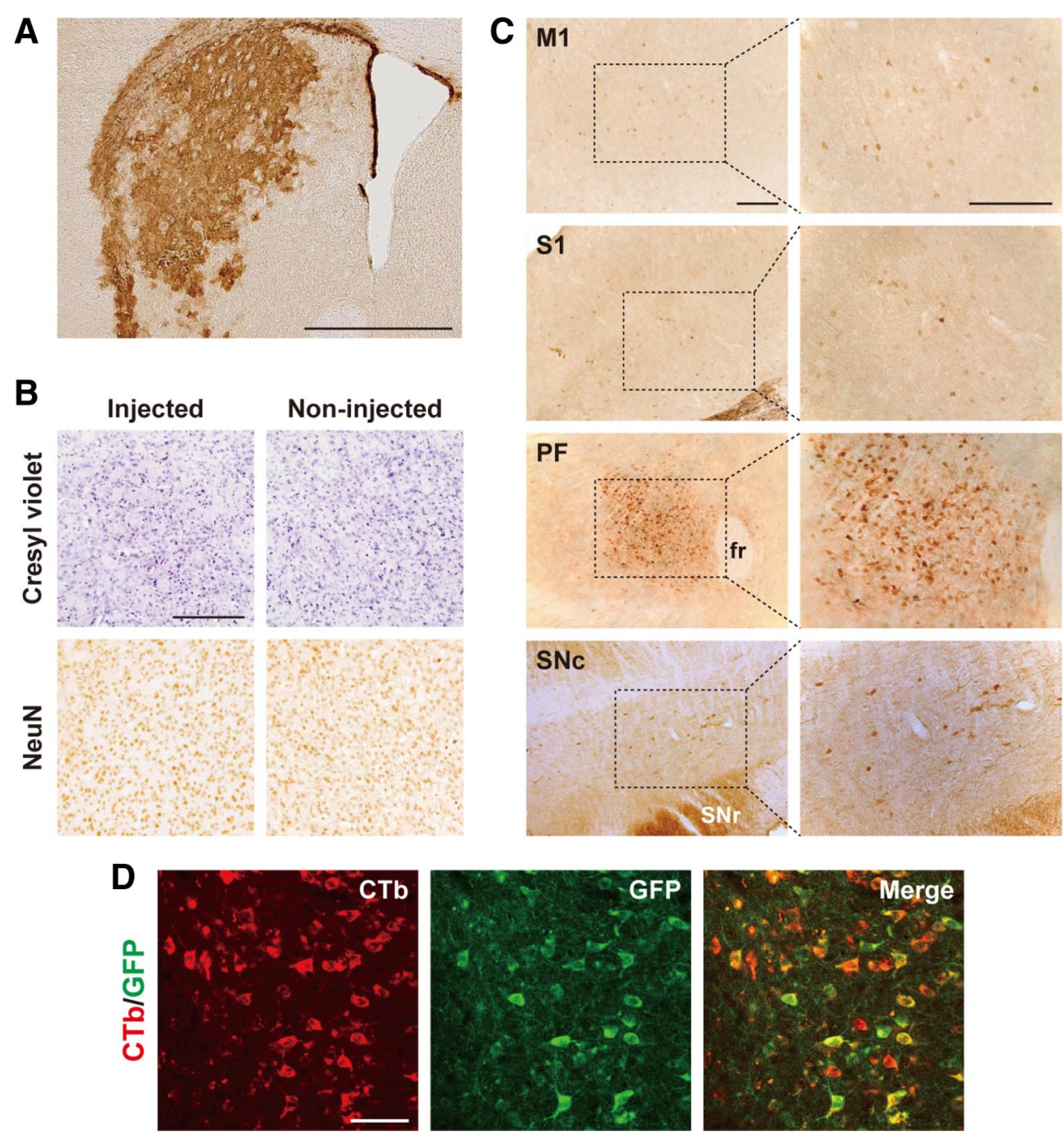

Figure 2. Expression of IL-2R $\alpha$-GFP transgene in neurons innervating the dorsolateral striatum. The HiRet vector encoding IL-2R $\alpha-G F P\left(2.0 \times 10^{9}\right.$ transduction units $\left./ \mathrm{ml}\right)$ was injected into the dorsal striatum of mice, and 5 weeks later their brains were processed and sections through the striatum were prepared and used for histological examination. $A$, GFP immunohistochemistry showing IL-2R $\alpha$-GFP expression in the dorsolateral striatum around the injection sites. B, Cresyl violet staining and NeuN immunostaining of sections on the injected and non-injected sides of the striatum. C, GFP immunohistochemistry demonstrating transgene expression through retrograde transport in the M1, S1, PF, and SNc. Right, Magnified views of the dotted rectangles in the left photos. D, Double fluorescence histochemistry with Alexa Fluor 555-conjugated cholera toxin subunit B (CTb) and immunostained GFP. Confocal microscopic images of PF sections are shown. (Tb-positive signals, GFP-positive signals, and merged images are indicated in red, green, and yellow, respectively. fr, Fasciculus retroflexus; SNr, substantia nigra pars reticulata. Scale bars: $\boldsymbol{A}, 1 \mathrm{~mm} ; \boldsymbol{B}, \boldsymbol{C}, 200$ $\mu \mathrm{m} ; \boldsymbol{D}, 50 \mu \mathrm{m}$.

METH-induced locomotor activity, one-way ANOVA). In addition, a previous study using excitotoxic lesions revealed that the dorsal striatum is necessary for the acquisition of motor skill learning (Yin et al., 2009). So we explored this type of learning by using an accelerating rotarod, and the time that each mouse maintained its balance on the rotating rod was measured as latency to fall (Fig. $4 B$ ). The latency gradually increased along with the trial number similarly among the HiRet-IL-2R $\alpha-$ GFP and HiRet-GFP vector-injected mice treated with IT or PBS (group effect, $F_{(3,27)}=0.03, p=0.99$; trial effect, $F_{(8,216)}=24.73, p<$ 0.001 ; group $\times$ trial interaction, $F_{(24,216)}=0.31, p=1.00$, ANOVA with repeated measures). Therefore, elimination of the thalamostriatal pathway in the HiRet-IL-2R $\alpha$-GFP vectorinjected group after IT treatment did not alter spontaneous and drug-induced locomotion or motor skill learning that require the dorsal striatum.

\section{Thalamostriatal ablation disturbs visual discrimination}

Excitotoxic lesions of neurons in the dorsal striatum impair the choice accuracy of motor responses and reduce the response rate in conditional discrimination (Brown and Robbins, 1989; Featherstone and McDonald, 2004, 2005). To address the roles of the thalamostriatal pathway in discrimination learning, we used a two-choice reaction time task dependent on visual stimulus (Lee et al., 2002). In this task, two levers were presented at the same time and one of the levers was randomly illuminated by a stimulus light. Animals learned to press the lever on the illuminated side within a $5 \mathrm{~s}$ test period after the onset of lever presentation contingent on the delivery of food pellets. First, we examined the behavioral consequence of selective elimination of the thalamostriatal pathway in the acquisition of this task. Mice were given an injection of the HiRet-IL-2R $\alpha-$ GFP or HiRet-GFP vector into their dorsolateral striatum and then injected with IT or PBS solution into their PF. On day 7 after the IT/PBS treatment, the mice started the conditioning schedule for the two-choice reaction time task. A lever press on the illuminated side within $5 \mathrm{~s}$ was defined as a "correct response," whereas a lever press on the side that was not illuminated was defined as an "error response." When the mice did not make a choice within $5 \mathrm{~s}$, the trial was counted as an "omission response." The correct response ratio 

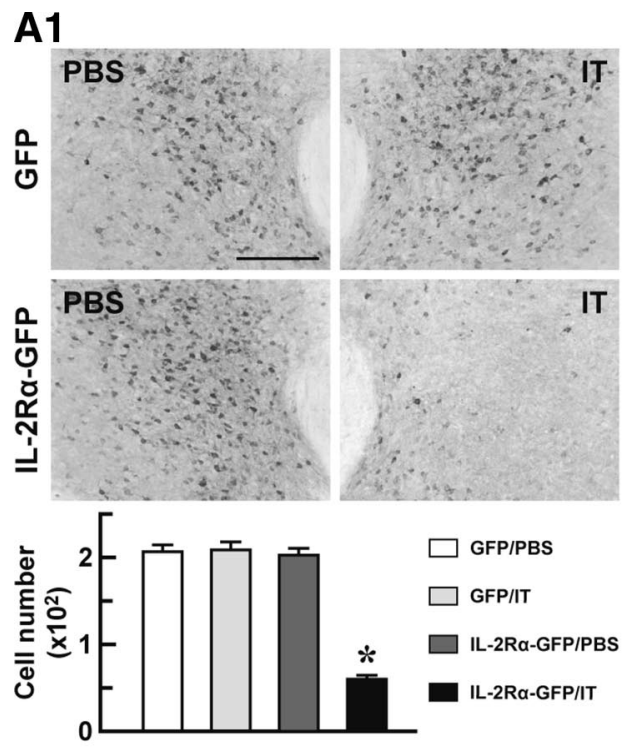

A2

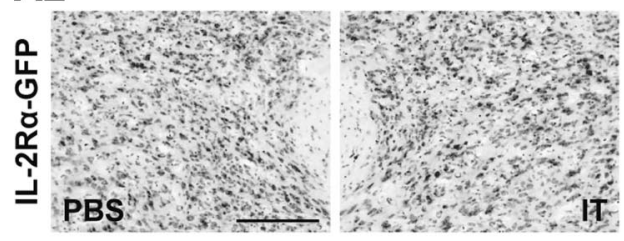

B
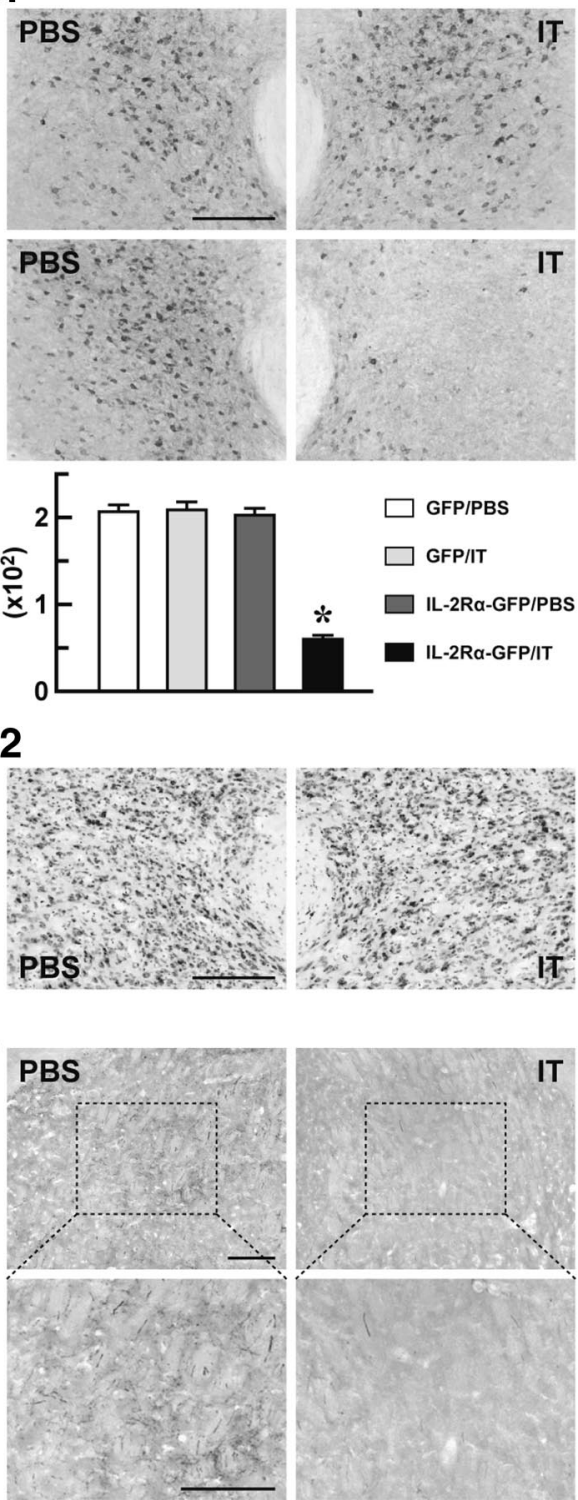

\section{(⿸丆口}
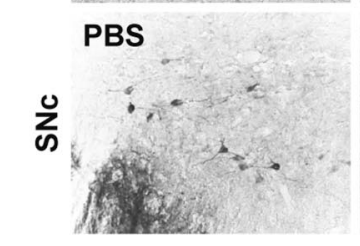

A4

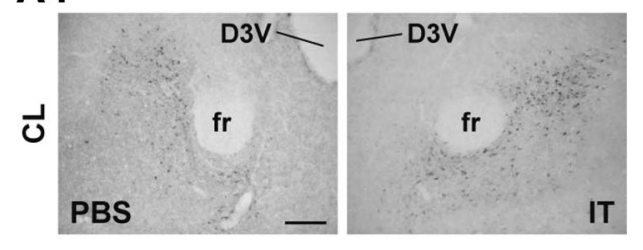

C

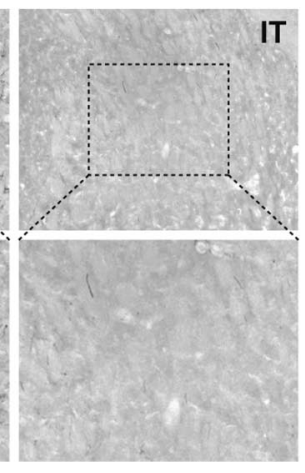

A3
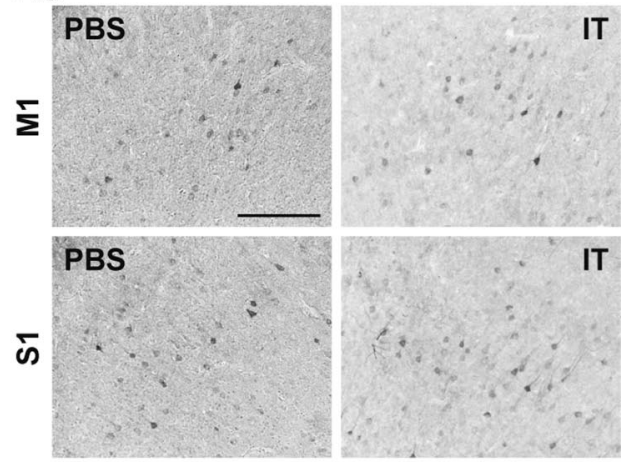

IT
IT

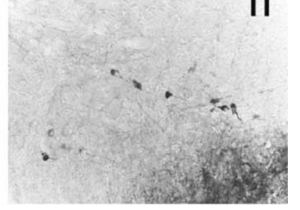

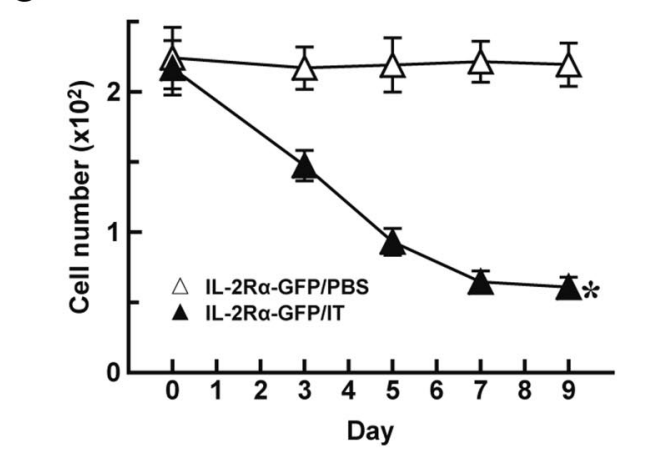

\section{.}

Figure 3. Selective elimination of PF neurons innervating the dorsolateral striatum. A, Impact of IT treatment on cells transduced retrogradely by the HiRet vector. Mice ( $n=4$ for each group) received a bilateral injection of the HiRet-IL-2R $\alpha$-GFP or HiRet-GFP vector into the dorsolateral striatum, and 5 weeks later they were treated with IT or PBS by injection into the unilateral PF. On day 7 after the treatment, brains were processed for histological examinations. GFP immunohistochemistry of sections through the PF $\left(A_{7}\right)$. The top shows representative images of GFP immunohistochemistry, and the bottom shows the number of GFP-positive cells per section. ${ }^{*} p<0.001$ compared with each of the HiRet-GFP/PBS, HiRet-GFP/IT, and HiRet-IL-2R $\alpha-G F P / P B S$ groups (Bonferroni's test). Cresyl violet staining of PF sections prepared from the HiRet-IL-2R $\alpha$-GFP-injected mice $\left(\boldsymbol{A}_{2}\right)$. GFP immunohistochemistry of M1, S1, and SNc sections prepared from the HiRet-IL-2R $\alpha$-GFP-injected mice $\left(\boldsymbol{A}_{3}\right)$. GFP immunostaining of CL sections prepared from the same animals $\left(\boldsymbol{A}_{4}\right)$. $\boldsymbol{B}$, Anterograde tracing of axons arising from the PF. The HiRet-IL-2R $\alpha-$ GFP vector-injected animals treated with IT or PBS by unilateral injection into the PF were used for BDA injection into the PF, and $7 \mathrm{~d}$ later their brains were processed. Sections through the dorsolateral striatum were prepared and stained for BDA. Bottom images are magnified views of the dotted rectangles in the top images. C, Time course of cell elimination. Mice ( $n=4$ for each group) were given a bilateral injection of the HiRet-IL-2R $\alpha$-GFP vector and treated unilaterally with IT or PBS into the PF. The brains were processed on different days after the treatment and stained by GFP immunohistochemistry. The number of GFP-positive cells per section was plotted. D3V, Dorsal third ventricle; fr, fasciculus retroflexus. ${ }^{*} p<0.001$ compared with the HiRet-IL-2R $\alpha-G F P / P B S$ group, Bonferroni's test. Scale bar, $200 \mu \mathrm{m}$.

was the percentage of the number of correct responses divided by the total number of correct and error responses, and the omission ratio was the percentage of the number of omission responses divided by the total trial number. The correct response ratio, correct response time, and omission ratio in each session block comprising five serial sessions were calculated. The correct response ratio was elevated along with increasing session blocks and showed a significant difference among the four mouse groups (Fig. 5A; group effect, $F_{(3,43)}=6.19, p<0.005$; session block effect, $F_{(9,387)}=42.1, p<0.001$; group $\times$ session block interaction, $F_{(27.387)}=2.49, p<0.001$, ANOVA with repeated measures). The elevation of the correct response ratio was remarkably impaired in the HiRet-IL-2R $\alpha$-GFP/IT group compared with each of the HiRet-GFP/PBS, HiRet-GFP/IT, and HiRet-IL-2R $\alpha-$ GFP/PBS groups ( $p<0.05$, Bonferroni's test). The correct response time also exhibited a significant difference among the four groups (Fig. $5 B$; group effect, $F_{(3,43)}=6.38, p<$ 0.005 ; session block effect, $F_{(9,387)}=1.79, p=0.07$; group $\times$ session block interaction, $F_{(27,387)}=1.01, p=0.45$, ANOVA with repeated measures), showing a moderate lengthening in the 

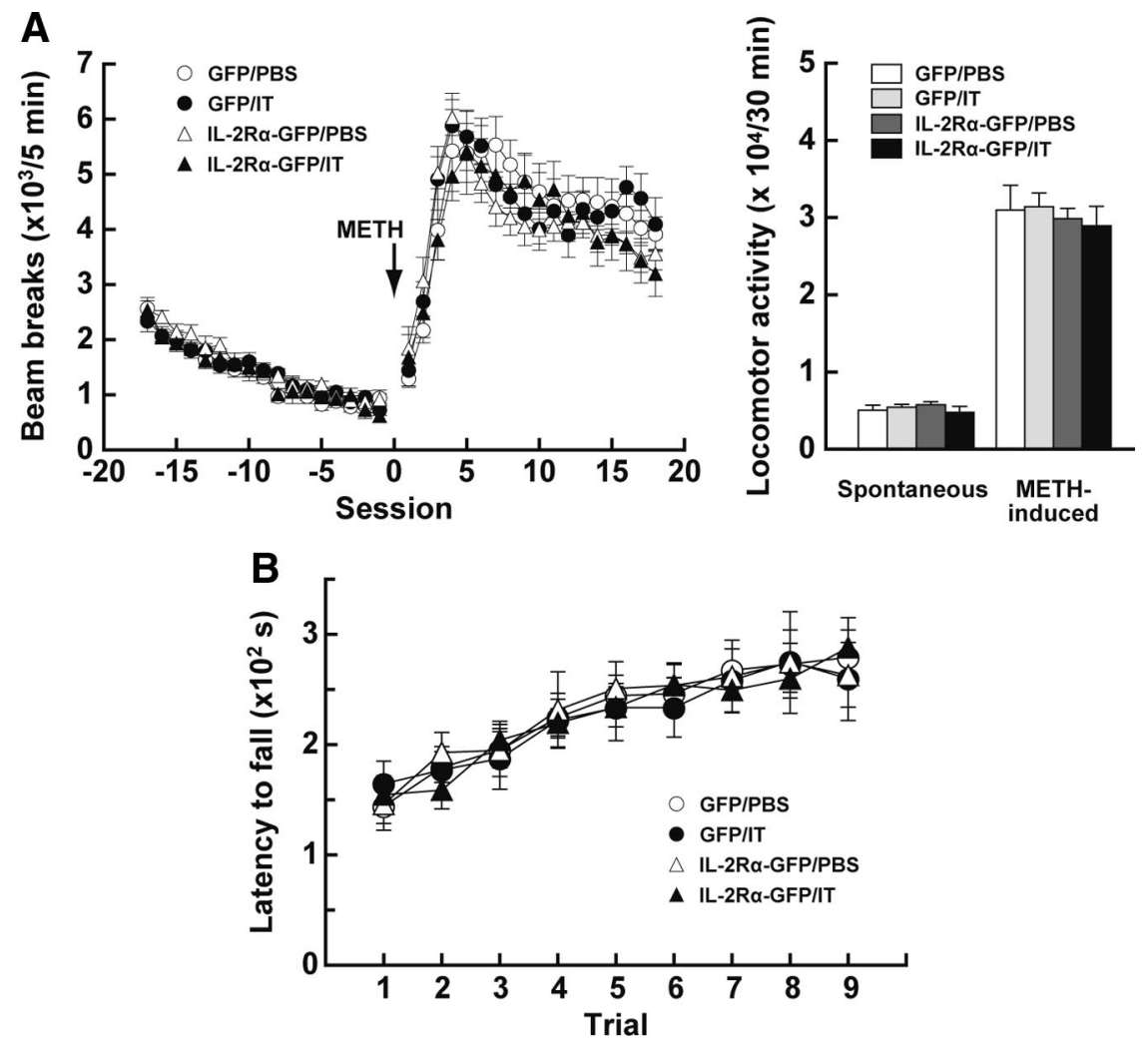

Figure 4. Locomotion and motor skill learning in mice lacking the PF-derived thalamostriatal pathway. $\boldsymbol{A}$, Spontaneous locomotion and drug-induced hyperactivity. Mice received a bilateral injection of the HiRet-IL-2R $\alpha$-GFP or HiRet-GFP vector into the striatum and then were injected bilaterally with IT or PBS into their PF ( $n=7$ for each group). After monitoring spontaneous locomotion for $1.5 \mathrm{~h}$, the mice were administered METH ( $4 \mathrm{mg} / \mathrm{kg}$, i.c.), and beam breaks over a 5 min session were enumerated (left graph). The total number of beam breaks in a 30 min test period was calculated to evaluate spontaneous locomotor activity during the pretreatment (sessions -6 to -1 ) and drug-induced locomotor activity after METH treatment (sessions 2-7; right graph). $\boldsymbol{B}$, Motor skill learning. Mice ( $n=8$ for each group) were given a bilateral injection of the HiRet-IL-2R $\alpha-$ GFP or HiRet-GFP vector into their striatum and then bilaterally injected with IT or PBS into their PF. The mice were trained on the accelerating rotarod, three trials per day, for 3 consecutive days, and the latency to fall was measured during each trial.

A

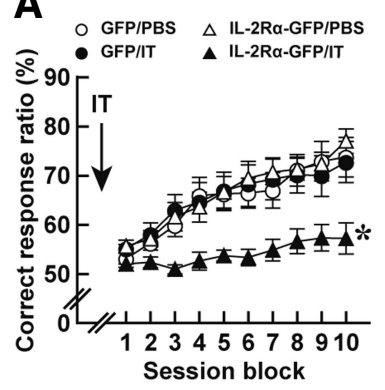

B

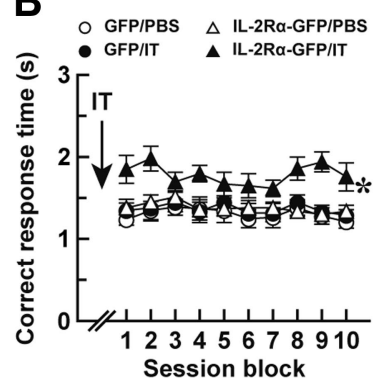

C

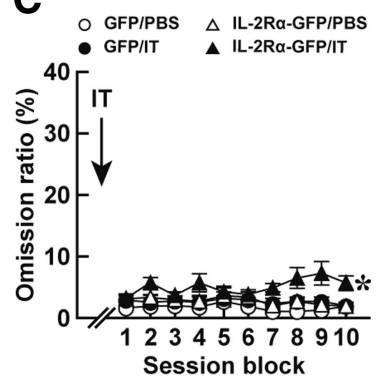

Figure 5. Impaired acquisition of visual discrimination in mice lacking the thalamostriatal pathway. Mice ( $n=11$ or $12 \mathrm{for}$ each group) received a bilateral injection of the HiRet-IL-2R $\alpha$-GFP or HiRet-GFP vector into their dorsolateral striatum, were bilaterally injected with IT or PBS into their PF, and were then tested in the two-choice reaction time task. The mice were presented with two levers and trained to press the lever on the light-illuminated side within $5 \mathrm{~s}$ after lever presentation. The correct response ratio $(\boldsymbol{A})$, correct response time $(\boldsymbol{B})$, and omission ratio $(\boldsymbol{C})$ in each session block comprising five continuous sessions are plotted. ${ }^{*} p<$ 0.05 compared with each of the HiRet-GFP/PBS, HiRet-GFP/IT, and HiRet-IL-2R $\alpha$-GFP/PBS groups, Bonferroni's test.

HiRet-IL-2R $\alpha-$ GFP/IT group compared with each of the three other groups ( $p<0.05$, Bonferroni's test.). In addition, the omission ratio also revealed a significant difference among the four groups (Fig. $5 C$; group effect, $F_{(3,43)}=6.62, p<0.005$; session block effect, $F_{(9,387)}=0.77, p=0.64$; group $\times$ session block interaction, $F_{(27,387)}=1.55, p<0.05$, ANOVA with repeated measures), being slightly but significantly increased in the HiRet-IL-2R $\alpha-$
GFP/IT group ( $p<0.05$, Bonferroni's test). These data indicate that the selective elimination of the thalamostriatal pathway impaired the accuracy of response selection and delayed the motor response during the formation of visual discrimination.

To explore the impact of thalamostriatal elimination on the performance of the visual two-choice reaction time task, we eliminated the pathway after the acquisition of the task. Mice given an injection of the HiRet-IL-2R $\alpha$-GFP or HiRet-GFP vector into their striatum were trained to reach an average of a correct response ratio of $>70 \%$ on 3 consecutive days. After achievement of this $70 \%$ criterion, the mice were injected with IT into the PF, and after $7 \mathrm{~d}$, they underwent the posttreatment training for $2 \mathrm{~d}$ and were then tested for the performance of the task. The correct response ratio, correct response time, and omission ratio in each session were counted. The correct response ratio was significantly different between the HiRet-GFP/IT and HiRet-IL-2R $\alpha-$ GFP/IT groups (Fig. 6A; group effect, $F_{(1,20)}=13.0, p<0.005$; session effect, $F_{(9,180)}=4.49, p<0.001$; group $\times$ session interaction, $F_{(9,180)}=2.75$, $p<0.01$, ANOVA with repeated measures), and the ratio was prominently lower in the HiRet-IL-2R $\alpha$-GFP vector-injected mice than in the HiRet-GFP vector-injected animals ( $p<0.005$, Bonferroni's test). In contrast, the correct response time was not altered between the HiRet-IL-2R $\alpha$-GFP and HiRet-GFP vector-injected mice (Fig. $6 B$; group effect, $F_{(1,20)}=1.01, p=0.33$; session effect, $F_{(9,180)}=1.57, p=0.13$; group $\times$ session interaction, $F_{(9,180)}=0.67$, $p=0.74$, ANOVA with repeated measures), and the omission ratio was indistinguishable between these two groups (Fig. 6C; group effect, $F_{(1,20)}=0.26, p=0.62$; session effect, $F_{(9,180)}=1.06, p=0.40$; group $\times$ session interaction, $F_{(9,180)}=0.97, p=0.47$, ANOVA with repeated measures). These data demonstrate that the elimination of the thalamostriatal pathway disturbed the accuracy of response selection in the execution of cued discrimination, although it did not substantially affect the response time or omission ratio.

The results obtained from the above behavioral studies with two strategies of the visual two-choice reaction time task demonstrated the contribution of the PF-derived thalamostriatal pathway to the control of response selection accuracy in both the acquisition and performance of visual discrimination. In contrast, the results indicated the requirement of the pathway for the regulation of the correct response time in the acquisition of the discrimination task but not in the performance of the task. The temporal difference in the pathway requirement suggests a stage-specific role of the 
thalamostriatal pathway in the modulation of the response time of learned motor actions.

Pathway ablation does not affect simple lever press and motivation

The response deficits in the visual discrimination task observed in the mice lacking the thalamostriatal pathway may be related to impairments in the simple lever-press response. To check this possibility, we performed a simple reaction time task. In this paradigm, one lever illuminated by the stimulus light was presented, and animals learned to press the lever for the food delivery within a $5 \mathrm{~s}$ test period after the initiation of lever presentation. Mice were given an injection of the HiRet-IL-2R $\alpha$-GFP or HiRet-GFP vector into their striatum and were then injected with IT into their PF. On day 7 after IT treatment, the mice began the conditioning schedule for the simple reaction time task. A lever press within $5 \mathrm{~s}$ was defined as a correct response. When the mice did not press the lever within $5 \mathrm{~s}$, the trial was counted as an omission response. The correct response ratio was the percentage of the number of correct responses divided by the total number of correct and omission responses. The response ratio showed a gradual elevation along with the increasing session numbers, and the values were indistinguishable between the HiRet-IL-2R $\alpha-$ GFP and HiRet-GFP vector-injected mice (Fig. $7 A_{1}$; group effect, $F_{(1,14)}=0.35, p=$ 0.56 ; session effect, $F_{(4,56)}=31.4, p<0.001$; group $\times$ session interaction, $F_{(4,56)}=0.21, p=0.93$, ANOVA with repeated measures). The correct response time was also unchanged between these two kinds of mouse groups (Fig. $7 A_{2}$; group effect, $F_{(1,14)}=$ $0.03, p=0.86$; session effect, $F_{(4,56)}=28.1, p<0.001$; group $\times$ session interaction, $F_{(4,56)}=0.40, p=0.81$, ANOVA with repeated measures). Therefore, the deletion of the thalamostriatal pathway did not influence the motor response without a visual discrimination process in the simple reaction time task, excluding the possibility that the deficits in the visual discrimination task caused by thalamostriatal ablation were associated with changes in the simple lever-press response.

In addition, we assessed the motivation of mice by using a progressive ratio task that measures the amount of effort that an animal continues to expend to obtain a reward. In this paradigm, mice were required to make a set number of lever pressings for food delivery. Two lever presses were demanded for the first trial, and the number of lever presses was doubled with each successive trial. The HiRet-IL-2R $\alpha-$ GFP or HiRet-GFP vector-injected mice were trained sequentially by the fixed ratio 1 and fixed ratio 4 schedules and injected with IT into their PF. After recovering for $7 \mathrm{~d}$, the mice started the progressive ratio task. The total number of lever presses or rewards during the session was comparable between the HiRet-IL-2R $\alpha-$ GFP and HiRet-GFP vector-injected animals (Fig. $7 B ; t_{(14)}=0.40, p=$ 0.70 for lever press; $t_{(14)}=0.67, p=0.51$ for reward). These data indicate that motivation of the mice lacking the thalamostriatal pathway was unaltered with respect to this measure, suggesting that the impairments of the formation and execution of visual discrimination did not stem from a changed motivational state in these animals.
B

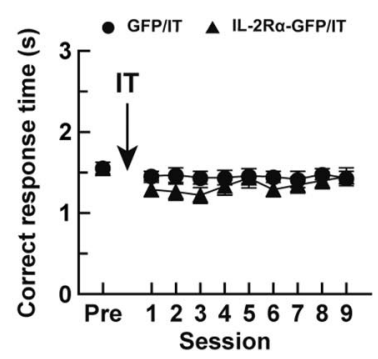

C

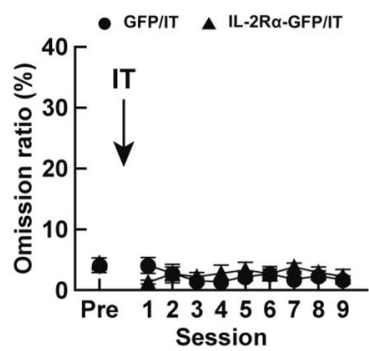

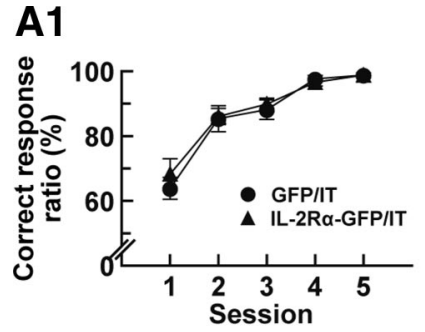

A2
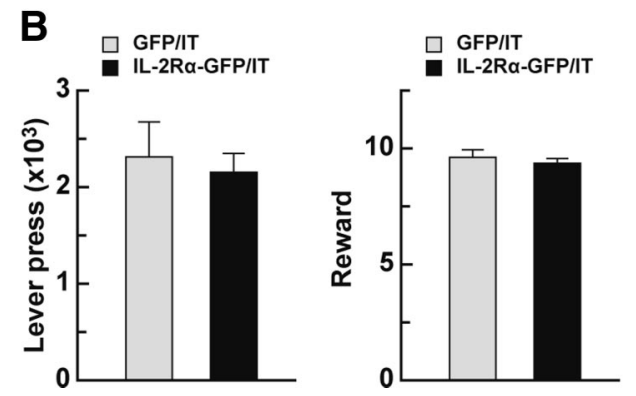

Figure 7. Simple lever-press response and motivation in animals with deleted thalamostriatal pathway. $\boldsymbol{A}$, Simple reaction time task. Mice ( $n=8$ for each group) received a bilateral injection of the HiRet-IL-2R $\alpha$-GFP or HiRet-GFP vector into the dorsolateral striatum and then were injected bilaterally with IT into their PF and tested for the simple reaction time task. The mice were trained to press the lever within a $5 \mathrm{~s}$ test period after the initiation of lever presentation. The correct response ratio $\left(A_{7}\right)$ and correct response time $\left(A_{2}\right)$ in each session are plotted.

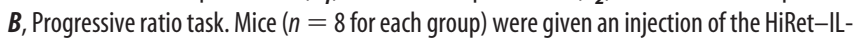
$2 R \alpha-G F P$ or HiRet-GFP vector into their striatum, treated with IT by injection into their PF, and tested by the progressive ratio program. The total number of lever presses or rewards was counted during the session.

\section{Discussion}

To investigate the behavioral roles of the thalamostriatal pathway in the striatum-dependent behaviors, we selectively removed the neural pathway arising from the PF and innervating the dorsolateral striatum in mice. The injection of the HiRet vector encoding IL-2R $\alpha-$ GFP into the dorsolateral striatum induced the transgene expression in the majority of PF neurons innervating the striatum. IT injection into the PF of the HiRet vector-injected mice resulted in a selective, efficient elimination of the thalamostriatal pathway derived from the PF. This elimination impaired the accuracy of response selection and delayed the motor response during the formation of a visual cue-dependent discriminative behavior. When the elimination was induced after the acquisition of discrimination, it also disturbed the response ac- 
curacy in the task performance with no apparent change in the response speed. The elimination did not influence spontaneous and drug-induced locomotion, motor skill learning, simple lever press, or motivational behavior. Together, these results highlight that the thalamostriatal projection derived from the PF plays important roles in the acquisition and execution of discrimination learning in response to a sensory stimulus.

Previous studies with excitotoxic lesions of the rat brain showed that the intralaminar thalamic nuclei are involved in several types of memory and learning paradigms, such as working memory, motor intention, and active avoidance (Burk and Mair, 1998; Massanés-Rotger et al., 1998; Mair et al., 2002). The dorsolateral and dorsomedial striatum appear to have different functions in these behavioral paradigms that involve the intralaminar thalamic nuclei (Mair et al., 2002). However, less is known about the behavioral roles of the thalamostriatal pathway originating from a particular intralaminar nuclear group and projecting to a subdivision of the striatum. The data of our present study provide direct evidence for the roles of the PF-derived thalamostriatal pathway innervating the dorsolateral striatum, showing the significance of this pathway in the control of sensory cue-dependent associative learning.

Selective elimination of the PF-derived thalamostriatal pathway did not alter spontaneous and drug-induced locomotion and the learning of motor skills that demand the function of the dorsal striatum (Costall et al., 1976; Coyle and Schwarcz, 1976; Yin et al., 2009). These results suggest that the thalamostriatal pathway is not implicated in these striatum-dependent behaviors. In contrast, the formation and maintenance of visual discrimination were susceptible to the ablation of the thalamostriatal pathway. These data suggest that this pathway may relay the information involved in the stimulus-response association by regulating striatal neuronal activity. The CM/PF neurons in monkeys are reported to be activated in response to a sensory stimulus that triggers attention and arousal (Matsumoto et al., 2001; Minamimoto and Kimura, 2002; Kimura et al., 2004). The thalamostriatal projection innervates two subpopulations of medium-sized spiny neurons constituting the direct and indirect pathways in rodents (Castle et al., 2005; Doig et al., 2010), whereas the afferents from the monkey CM/PF neurons appear to predominantly innervate the direct pathway neurons (Sidibé and Smith, 1996). The in vivo stimulation of thalamic neurons elicits excitatory responses of striatal medium spiny neurons (Kocsis et al., 1977; Vandermaelen and Kitai, 1980), and in vitro electrophysiological analysis of transgenic mice shows that both the direct and indirect pathway neurons respond to thalamic stimulation (Ding et al., 2008). In addition, the thalamostriatal projection makes synaptic contacts with striatal cholinergic interneurons (Lapper and Bolam, 1992), and electrical stimulation of thalamic neurons facilitates acetylcholine release in the striatum (Consolo et al., 1996). The examination of slice recordings shows that activation of thalamostriatal neurons induces a burst of spikes in cholinergic interneurons and thereby suppresses corticostriatal inputs to medium spiny neurons through a presynaptic mechanism (Ding et al., 2010). Furthermore, the nigrostriatal dopamine neurons projecting to the dorsal striatum subserve the acquisition of conditional discrimination (Robbins et al., 1990), and these dopamine neurons are activated by a sensory cue that predicts reward (Schultz and Romo, 1990; Ljungberg et al., 1991, 1992; Kawagoe et al., 2004). These data suggest that the stimulus-induced activation of thalamostriatal neurons, in conjunction with the corticostriatal or nigrostriatal system, may contribute to a synaptic mechanism by which striatal spiny neurons and cholinergic interneurons undergo plastic changes requisite for the formation of learning.

The elimination of the PF-derived thalamostriatal pathway, when induced after the acquisition of visual discrimination, abolished the response accuracy in the task performance but did not produce any change in the correct response time. The influence on the response time was in contrast to the prolonged motor response observed in the IT-injected animals when they were tested for the acquisition of discrimination learning. This distinction in the response time effect indicates that the thalamostriatal pathway is required for regulating the response time during the development of visual discrimination and suggests that the role of the thalamostriatal pathway, after learning acquisition, may be transferred to other neural systems. Recent studies with functional magnetic resonance imaging indicate that neural activity linked to motor learning undergoes dynamic shifts in some brain regions, including the corticostriatal system, and that these shifts vary depending on the stage of learning (Doyon et al., 2003; Parsons et al., 2005). Indeed, both in vitro and in vivo electrophysiological studies show the development of long-term plasticity at the corticostriatal synapses (Calabresi et al., 1992; Charpier and Deniau, 1997; Fino et al., 2005; Di Filippo et al., 2009). Thus, striatal input pathways from some cortical areas may replace the function of the thalamostriatal pathway to facilitate motor responses after the repetitive training of the task.

We used the HiRet vector to express the receptor for the recombinant IT in neurons through retrograde transport of the vector. The injection of IT into a specific brain region of the HiRet vector-injected animals eliminated selective neural pathways from the complex neural circuitry. This technology for pathway-specific targeting was successfully applied for the study of behavioral roles of the thalamostriatal pathway arising from the PF and projecting to the dorsolateral striatum. By changing the injection sites of the HiRet vector or IT to other ones, we will be able to examine the behavioral roles of other neural pathways. Subsequent applications should be to target the neural pathways originating from other intralaminar nuclear groups projecting to the striatal subdivisions or the corticostriatal inputs derived from different areas in the cerebral cortex. Our technology will provide a general, powerful strategy to investigate not only the exact roles of specific neural pathways constituting the neural circuitry but also the mechanisms underlying a wide range of brain functions on the basis of the decoding of the neural network.

\section{References}

Alexander GE, Crutcher MD (1990) Functional architecture of basal ganglia circuits: neural substrates of parallel processing. Trends Neurosci 13:266-271.

Balleine BW, Liljeholm M, Ostlund SB (2009) The integrative function of the basal ganglia in instrumental conditioning. Behav Brain Res 199:43-52.

Berendse HW, Groenewegen HJ (1990) Organization of the thalamostriatal projections in the rat, with special emphasis on the ventral striatum. J Comp Neurol 299:187-228.

Brown VJ, Robbins TW (1989) Elementary processes of response selection mediated by distinct regions of the striatum. J Neurosci 9:3760-3765.

Burk JA, Mair RG (1998) Thalamic amnesia reconsidered: excitotoxic lesions of the intralaminar nuclei, but not the mediodorsal nucleus, disrupt place delayed matching-to-sample performance in rats (Rattus norvegicus). Behav Neurosci 112:54-67.

Calabresi P, Maj R, Pisani A, Mercuri NB, Bernardi G (1992) Long-term synaptic depression in the striatum: physiological and pharmacological characterization. J Neurosci 12:4224-4233.

Castle M, Aymerich MS, Sanchez-Escobar C, Gonzalo N, Obeso JA, Lanciego JL (2005) Thalamic innervation of the direct and indirect basal ganglia 
pathways in the rat: ipsi- and contralateral projections. J Comp Neurol 483:143-153.

Charpier S, Deniau JM (1997) In vivo activity-dependent plasticity at cortico-striatal connections: evidence for physiological long-term potentiation. Proc Natl Acad Sci U S A 94:7036-7040.

Consolo S, Baldi G, Giorgi S, Nannini L (1996) The cerebral cortex and parafascicular thalamic nucleus facilitate in vivo acetylcholine release in the rat striatum through distinct glutamate receptor subtypes. Eur J Neurosci 8:2702-2710.

Costall B, Marsden CD, Naylor RJ, Pycock CJ (1976) The relationship between striatal and mesolimbic dopamine dysfunction and the nature of circling responses following 6-hydroxydopamine and electrolytic lesions of the ascending dopamine systems of rat brain. Brain Res 118:87-113.

Coyle JT, Schwarcz R (1976) Lesion of striatal neurones with kainic acid provides a model for Huntington's chorea. Nature 263:244-246.

DeLong MR (1990) Primate models of movement disorders of basal ganglia origin. Trends Neurosci 13:281-285.

Di Filippo M, Picconi B, Tantucci M, Ghiglieri V, Bagetta V, Sgobio C, Tozzi A, Parnetti L, Calabresi P (2009) Short-term and long-term plasticity at corticostriatal synapses: implications for learning and memory. Behav Brain Res 199:108-118.

Ding J, Peterson JD, Surmeier DJ (2008) Corticostriatal and thalamostriatal synapses have distinctive properties. J Neurosci 28:6483-6492.

Ding JB, Guzman JN, Peterson JD, Goldberg JA, Surmeier DJ (2010) Thalamic gating of corticostriatal signaling by cholinergic interneurons. Neuron 67:294-307.

Doig NM, Moss J, Bolam JP (2010) Cortical and thalamic innervation of direct and indirect pathway medium-sized spiny neurons in mouse striatum. J Neurosci 30:14610-14618.

Doyon J, Penhune V, Ungerleider LG (2003) Distinct contribution of the cortico-striatal and cortico-cerebellar systems to motor skill learning. Neuropsychologia 41:252-262.

Doyon J, Bellec P, Amsel R, Penhune V, Monchi O, Carrier J, Lehéricy S, Benali H (2009) Contributions of the basal ganglia and functionally related brain structures to motor learning. Behav Brain Res 199:61-75.

Featherstone RE, McDonald RJ (2004) Dorsal striatum and stimulusresponse learning: lesions of the dorsolateral, but not dorsomedial, striatum impair acquisition of a stimulus-response-based instrumental discrimination task, while sparing conditioned place preference learning. Neuroscience 124:23-31.

Featherstone RE, McDonald RJ (2005) Lesions of the dorsolateral striatum impair the acquisition of a simplified stimulus-response dependent conditional discrimination task. Neuroscience 136:387-395.

Fino E, Glowinski J, Venance L (2005) Bidirectional activity-dependent plasticity at corticostriatal synapses. J Neurosci 25:11279-11287.

François C, Percheron G, Parent A, Sadikot AF, Fenelon G, Yelnik J (1991) Topography of the projection from the central complex of the thalamus to the sensorimotor striatal territory in monkeys. J Comp Neurol 305:17-34.

Graybiel AM (2005) The basal ganglia: learning new tricks and loving it. Curr Opin Neurobiol 15:638-644.

Hanawa H, Kelly PF, Nathwani AC, Persons DA, Vandergriff JA, Hargrove P, Vanin EF, Nienhuis AW (2002) Comparison of various envelope proteins for their ability to pseudotype lentiviral vectors and transduce primitive hematopoietic cells from human blood. Mol Ther 5:242-251.

Hanawa H, Hematti P, Keyvanfar K, Metzger ME, Krouse A, Donahue RE, Kepes S, Gray J, Dunbar CE, Persons DA, Nienhuis AW (2004) Efficient gene transfer into rhesus repopulating hematopoietic stem cells using a simian immunodeficiency virus-based lentiviral vector system. Blood 103:4062-4069.

Hikosaka O, Nakamura K, Sakai K, Nakahara H (2002) Central mechanisms of motor skill learning. Curr Opin Neurobiol 12:217-222.

Jog MS, Kubota Y, Connolly CI, Hillegaart V, Graybiel AM (1999) Building neural representations of habits. Science 286:1745-1749.

Kato S, Kobayashi K, Inoue K, Kuramochi M, Okada T, Yaginuma H, Morimoto K, Shimada T, Takada M, Kobayashi K (2011) A lentiviral strategy for highly efficient retrograde gene transfer by pseudotyping with fusion envelope glycoprotein. Hum Gene Ther 22:197-206.

Kawagoe R, Takikawa Y, Hikosaka O (2004) Reward-predicting activity of dopamine and caudate neurons: a possible mechanism of motivational control of saccadic eye movement. J Neurophysiol 91:1013-1024.

Kimura M, Minamimoto T, Matsumoto N, Hori Y (2004) Monitoring and switching of cortico-basal ganglia loop functions by the thalamo-striatal system. Neurosci Res 48:355-360.

Knowlton BJ, Mangels JA, Squire LR (1996) A neostriatal habit learning system in humans. Science 273:1399-1402.

Kobayashi K, Morita S, Sawada H, Mizuguchi T, Yamada K, Nagatsu I, Fujita K, Kreitman RJ, Pastan I, Nagatsu T (1995) Immunotoxin-mediated conditional disruption of specific neurons in transgenic mice. Proc Natl Acad Sci U S A 92:1132-1136.

Kocsis JD, Sugimori M, Kitai ST (1977) Convergence of excitatory synaptic inputs to caudate spiny neurons. Brain Res 124:403-413.

Kreitman RJ, Bailon P, Chaudhary VK, FitzGerald DJ, Pastan I (1994) Recombinant immunotoxins containing anti- $\mathrm{Tac}(\mathrm{Fv})$ and derivatives of Pseudomonas exotoxin produce complete regression in mice of an interleukin-2 receptor-bearing human carcinoma. Blood 83:426-434.

Lapper SR, Bolam JP (1992) Input from the frontal cortex and the parafascicular nucleus to cholinergic interneurons in the dorsal striatum of the rat. Neuroscience 51:533-545.

Lee B, Tumu P, Paul IA (2002) Effects of LP-BM5 murine leukemia virus infection on errors and response time in a two-choice serial reaction time task in C57BL/6 mice. Brain Res 948:1-7.

Ljungberg T, Apicella P, Schultz W (1991) Responses of monkey midbrain dopamine neurons during delayed alternation performance. Brain Res 567:337-341.

Ljungberg T, Apicella P, Schultz W (1992) Responses of monkey dopamine neurons during learning of behavioral reactions. J Neurophysiol 67:145-163.

Mair RG, Koch JK, Newman JB, Howard JR, Burk JA (2002) A double dissociation within striatum between serial reaction time and radial maze delayed nonmatching performance in rats. J Neurosci 22:6756-6765.

Massanés-Rotger E, Aldavert-Vera L, Segura-Torres P, Martí-Nicolovius M, Morgado-Bernal I (1998) Involvement of the parafascicular nucleus in the facilitative effect of intracranial self-stimulation on active avoidance in rats. Brain Res 808:220-231.

Matsumoto N, Minamimoto T, Graybiel AM, Kimura M (2001) Neurons in the thalamic CM-Pf complex supply striatal neurons with information about behaviorally significant sensory events. J Neurophysiol 85:960-976.

Minamimoto T, Kimura M (2002) Participation of the thalamic CM-Pf complex in attentional orienting. J Neurophysiol 87:3090-3101.

Packard MG, Knowlton BJ (2002) Learning and memory functions of the basal ganglia. Annu Rev Neurosci 25:563-593.

Parent A, Hazrati LN (1995) Functional anatomy of the basal ganglia. I. The cortico-basal ganglia-thalamo-cortical loop. Brain Res Rev 20:91-127.

Parsons MW, Harrington DL, Rao SM (2005) Distinct neural systems underlie learning visuomotor and spatial representations of motor skills. Hum Brain Mapp 24:229-247.

Pasupathy A, Miller EK (2005) Different time courses of learning-related activity in the prefrontal cortex and striatum. Nature 433:873-876.

Paxinos G, Franklin KBJ (2001) The mouse brain in stereotaxic coordinates, Ed 2. San Diego: Academic.

Robbins TW, Giardini V, Jones GH, Reading P, Sahakian BJ (1990) Effects of dopamine depletion from the caudate-putamen and nucleus accumbens septi on the acquisition and performance of a conditional discrimination task. Behav Brain Res 38:243-261.

Rogers RD, Baunez C, Everitt BJ, Robbins TW (2001) Lesions of the medial and lateral striatum in the rat produce differential deficits in attentional performance. Behav Neurosci 115:799-811.

Sano H, Yasoshima Y, Matsushita N, Kaneko T, Kohno K, Pastan I, Kobayashi K (2003) Conditional ablation of striatal neuronal types containing dopamine $\mathrm{D}_{2}$ receptor disturbs coordination of basal ganglia function. J Neurosci 23:9078-9088.

Schultz W, Romo R (1990) Dopamine neurons of the monkey midbrain: contingencies of responses to stimuli eliciting immediate behavioral reactions. J Neurophysiol 63:607-624.

Sidibé M, Smith Y (1996) Differential synaptic innervation of striatofugal neurones projecting to the internal or external segments of the globus pallidus by thalamic afferents in the squirrel monkey. J Comp Neurol 365:445-465.

Smith Y, Raju DV, Pare JF, Sidibe M (2004) The thalamostriatal system: a highly specific network of the basal ganglia circuitry. Trends Neurosci 27:520-527. 
Smith Y, Raju D, Nanda B, Pare JF, Galvan A, Wichmann T (2009) The thalamostriatal systems: anatomical and functional organization in normal and parkinsonian states. Brain Res Bull 78:60-68.

Tremblay L, Hollerman JR, Schultz W (1998) Modifications of reward expectation-related neuronal activity during learning in primate striatum. J Neurophysiol 80:964-977.

Vandermaelen CP, Kitai ST (1980) Intracellular analysis of synaptic potentials in rat neostriatum following stimulation of the cerebral cortex, thalamus, and substantia nigra. Brain Res Bull 5:725-733.

Yasoshima Y, Kai N, Yoshida S, Shiosaka S, Koyama Y, Kayama Y, Kobayashi K (2005) Subthalamic neurons coordinate basal ganglia function through differential neural pathways. J Neurosci 25:7743-7753.
Yin HH (2010) The sensorimotor striatum is necessary for serial order learning. J Neurosci 30:14719-14723.

Yin HH, Knowlton BJ, Balleine BW (2004) Lesions of dorsolateral striatum preserve outcome expectancy but disrupt habit formation in instrumental learning. Eur J Neurosci 19:181-189.

Yin HH, Knowlton BJ, Balleine BW (2006) Inactivation of dorsolateral striatum enhances sensitivity to changes in the action-outcome contingency in instrumental conditioning. Behav Brain Res 166:189-196.

Yin HH, Mulcare SP, Hilário MR, Clouse E, Holloway T, Davis MI, Hansson AC, Lovinger DM, Costa RM (2009) Dynamic reorganization of striatal circuits during the acquisition and consolidation of a skill. Nat Neurosci $12: 333-341$ 